\title{
$X$-ray absorption and $x$-ray magnetic circular dichroism in bulk and thin films of ferrimagnetic $\mathrm{GdTiO}_{3}$
}

\author{
R. Aeschlimann, ${ }^{1}$ M. N. Grisolia, ${ }^{1}$ G. Sanchez-Santolino $\odot,{ }^{2}$ J. Varignon, ${ }^{1,3}$ F. Choueikani $\odot,{ }^{4}$ R. Mattana, ${ }^{1}$ \\ V. Garcia ${ }^{1}{ }^{1}$ S. Fusil, ${ }^{1}$ T. Fröhlich, ${ }^{5}$ M. Braden $\odot,{ }^{5}$ B. Delley, ${ }^{6}$ M. Varela, ${ }^{2}$ P. Ohresser, ${ }^{4}$ J. Santamaria, ${ }^{2}$ \\ A. Barthélémy, ${ }^{1}$ C. Piamonteze ${ }^{6}$ and M. Bibes ${ }^{1}{ }^{1, *}$ \\ ${ }^{1}$ Unité Mixte de Physique, CNRS, Thales, Université Paris-Saclay, 91767 Palaiseau, France \\ ${ }^{2}$ Facultad de CC. Físicas \& Instituto Pluridisciplinar, Universidad Complutense de Madrid, 28040 Madrid, Spain \\ ${ }^{3}$ Laboratoire de Cristallographie et Sciences des Matériaux, UMR6508 CNRS, ENSICAEN, Université de Caen-Basse Normandie, \\ 6 bd Maréchal Juin, 14050 Caen, France \\ ${ }^{4}$ Synchrotron SOLEIL, L'Orme des Merisiers Saint-Aubin, BP 48, 91192 Gif-sur-Yvette, France \\ ${ }^{5}$ II. Physikalisches Institut, Universität zu Köln, Zülpicher Str. 77, D-50937 Köln, Germany \\ ${ }^{6}$ Swiss Light Source, Paul Scherrer Institut, CH-5232 Villigen PSI, Switzerland
}

(Received 5 October 2020; accepted 16 December 2020; published 13 January 2021)

\begin{abstract}
Perovskite rare-earth titanates are prototypical Mott insulators in which $\mathrm{Ti}^{3+}$ ions with $3 d^{1}$ electronic configuration exhibit ferromagnetic or antiferromagnetic spin order, depending on the rare-earth size. This peculiar magnetic behavior has, however, been barely studied with element-specific probes, either in bulk or in thin films. The recent finding of fingerprints of ferromagnetism in two-dimensional electron gases at oxide interfaces involving rare-earth titanates has produced a surge of the interest in these complex materials. Harnessing the interfacial magnetic states in these heterostructures calls for a better understanding of their insufficiently explored magnetic states in bulk and especially in thin film form. In this paper, we combine high-resolution transmission electron microscopy with $\mathrm{x}$-ray absorption spectroscopy and $\mathrm{x}$-ray magnetic circular dichroism (XMCD) to determine the structural, electronic, and magnetic structure of $\mathrm{GdTiO}_{3}$ in bulk and thin film form. In both cases, we find that the sample surface is strongly overoxidized but a few $\mathrm{nm}$ below, $\mathrm{Ti}$ is mostly $3+$ and shows a large $\mathrm{XMCD}$. We provide evidence for the ferrimagnetic nature of $\mathrm{GdTiO}_{3}$ with antialigned $\mathrm{Gd}$ and Ti sublattices and show that, just as in antiferromagnetic $\mathrm{LaTiO}_{3}$ or ferromagnetic $\mathrm{YTiO}_{3}$, Ti carries no orbital moment.
\end{abstract}

DOI: 10.1103/PhysRevMaterials.5.014407

\section{INTRODUCTION}

The complexity of transition metal oxides results from the delicate interplay between lattice, charge, spin and orbital degrees of freedom. Due to the quenching of the orbital moment by the octahedral crystal field, magnetic interactions are usually spin-spin only, as formalized in the GoodenoughKanamori-Anderson (GKA) framework. In short, GKA rules are based on the assumption that the orientation of orbitals on neighboring sites controls the exchange interaction between magnetic ions [1]. While most experiments on transition metal oxides are in accordance with this paradigm, it has been called into question by some studies [2,3] in the prototypical Mott insulator family, $R \mathrm{TiO}_{3}(R \mathrm{TO})$, where $R$ stands for elements in the lanthanide row (and yttrium). In this $3 d^{1}$ system both spins and $t_{2 g}$ orbitals can display coupled long-range orders $[2,4]$, resulting in rich phase diagrams. Through a continuous change of the rare-earth ion size across the lanthanide series, the $R$ TO family displays a unique phase transition from an antiferromagnetic (AFM) spin order for large rare-earths $(R=\mathrm{La} \ldots \mathrm{Sm})$ to a ferromagnetic $(\mathrm{FM})$ spin order for relatively small rare earths $(R=\mathrm{Gd} \ldots \mathrm{Lu}$ or $R=\mathrm{Y})$ [2,5-9].

\footnotetext{
*manuel.bibes@cnrs-thales.fr
}

In parallel, the orbital structure is also modified across this transition [10].

The origin of the various spin-orbital orderings of RTO compounds as a function of the rare-earth radius has attracted a lot of interest since the 2000s. The mechanism was identified recently and simply relies on structural deformations associated with steric effects that are generic to any orthorhombic perovskite $[11,12]$. Due to $A$-to- $B$ cation size mismatch, quantified by Goldschmidt's tolerance factor [13], the symmetry is lowered from cubic $P m-3 m$ to orthorhombic Pbnm through the appearance of a $a^{-} a^{-} c^{+}$octahedra rotation pattern whose amplitude increases when going from $\mathrm{La}$ to $\mathrm{Y}$. The point group symmetry is thus lowered from $O_{\mathrm{h}}$ (cubic cell) to $D_{2 \mathrm{~h}}$ (orthorhombic cell) and a new basis of orbitals is created. As a result, the $\mathrm{Ti}^{3+} d$ electron sits in a $\alpha d_{\mathrm{xy}}+\beta d_{\mathrm{xz}}+\gamma d_{\mathrm{yz}}$ orbital $\left(\alpha^{2}+\beta^{2}+\gamma^{2}=1\right)$ that is a combination of the initial $t_{2 g}$ levels. The $\alpha, \beta$, and $\gamma$ coefficients are simply controlled by two antagonist antipolar $A_{\mathrm{R}}$ and $A_{\mathrm{X}}$ distortions (see Fig. 1 in Ref. [11]) involving rare-earth motions whose amplitudes are triggered by two lattice mode couplings with $\mathrm{O}_{6}$ rotations. Thereby, the $A_{\mathrm{X}}$ and $A_{\mathrm{R}}$ modes drive two very different occupied orbital shapes as a function of the rare-earth radius, resembling a balanced combination of the three initial $t_{2 \mathrm{~g}}$ levels: a balanced combination for compounds with low tolerance factor such as $\mathrm{YTiO}_{3}$, i.e., $\left[0.69 d_{x y}+0.73\left(\beta d_{x z}+\right.\right.$ 
$\left.\left.\gamma d_{y z}\right)\right]\left(A_{\mathrm{R}}\right.$ dominates) or a dominant $\left(\beta d_{x z}+\gamma d_{y z}\right)$ contribution for compounds with larger tolerance factor such as $\mathrm{LaTiO}_{3}$, i.e., $\left[0.57 d_{x y}+0.83\left(\beta d_{x z}+\gamma d_{y z}\right)\right]$ ( $A_{X}$ dominates). In turn, these two orbital shapes drive the magnetic interactions between nearest-neighbor $\mathrm{Ti}^{3+}$ spins and explain the transition from FM (Y-Gd) to G-type AFM orders (Sm-La) upon decreasing the octahedra rotation amplitude [14].

A substantial effort $[2,10,15-19]$ has also been made to measure experimentally the magnetic moments of the titanates and to understand their orbital properties. In $\mathrm{LaTiO}_{3}$, the spin moment was estimated to $0.46 \mu_{\mathrm{B}}[20,21]$ and $0.57 \mu_{\mathrm{B}}$ [8], which is smaller than both the value of $1 \mu_{\mathrm{B}}$ expected for a single electron with a quenched orbital moment, and a moment of $0.72 \mu_{\mathrm{B}}$, expected when taking quantum fluctuations into account [8]. A similar observation was made for $\mathrm{YTiO}_{3}$ using neutron polarized diffraction $\left(\sim 0.6 \mu_{\mathrm{B}}\right)$ [8] and superconducting quantum interference device (SQUID) magnetometry $\left(0.84 \mu_{\mathrm{B}}\right)$ [22]. Although the presence of an antiparallel orbital moment is sometimes proposed to explain the discrepancy between expected (i.e., $1 \mu_{\mathrm{B}}$ ) and measured magnetic moments [22], no orbital moment was observed in $\mathrm{YTiO}_{3}$ or in $\mathrm{LaTiO}_{3}$ [19,23-25], which can be explained by strong crystal electric fields suppressing the impact of spin orbit coupling [15]. Most studies so far were conducted on RTO compounds with nonmagnetic R ion such as $\mathrm{Y}$ and $\mathrm{La}$ [23-25]. Surprisingly, direct and simultaneous experimental measurements of orbital and spin moments in other RTO compounds have been missing. However, materials near the boundary between antiferromagnetic and ferromagnetic ordering of Ti moments seem the most interesting, because suppressed crystal fields may strengthen the impact of spin orbit coupling.

In parallel to their fundamental interest in bulk form, RTO compounds have also emerged as interesting building blocks to design novel states of matter at interfaces. For instance, $\mathrm{GdTiO}_{3}$ has been combined with $\mathrm{SrTiO}_{3}$ to define correlated oxide quantum wells [26] showing signs of ferromagnetism [27], and $\delta$-doped $\mathrm{GdTiO}_{3}$ heterostructures behave as a twodimensional small polaron gas [28]. At interfaces with various rare-earth nickelates, $\mathrm{GdTiO}_{3}$ was also found to induce a peculiar magnetic state in the nickelate, associated with electron transfer across the interface [29]. Other titanates such as $\mathrm{NdTiO}_{3}, \mathrm{SmTiO}_{3}$, and $\mathrm{YTiO}_{3}$ also lead to interfacial electron gases, when combined with $\mathrm{SrTiO}_{3}$ [30-32]. Despite this recent development of RTO thin film heterostructures, very few quantitative magnetic measurements have been made to complement the body of results on bulk specimens. Harnessing the interfacial magnetic states in heterostructures involving rare-earth titanates calls for a better understanding of their insufficiently explored magnetic states in bulk and especially in thin film form.

Here we report structural, magnetic, and electronic properties of bulk and thin film samples of $\mathrm{GdTiO}_{3}$, which is ferromagnetic with a $T_{\mathrm{C}}$ of $32 \mathrm{~K}$ [2], but sits at the border of the AFM to FM transition. We make use of $x$-ray absorption spectroscopy (XAS) combined with x-ray magnetic circular dichroism (XMCD) and electron energy-loss spectroscopy (EELS) as well as first-principles simulations to characterize the valence of the Ti ions and discuss the possible presence of an orbital moment on Ti. (a)

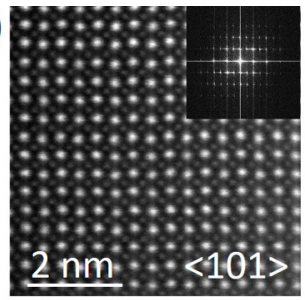

(c)

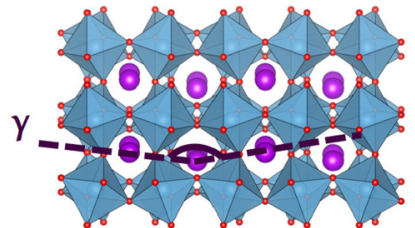
$<101>$ (b)

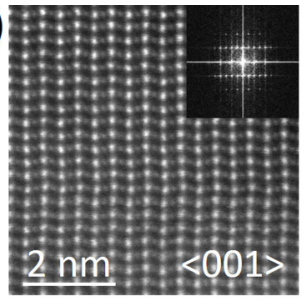

(d)

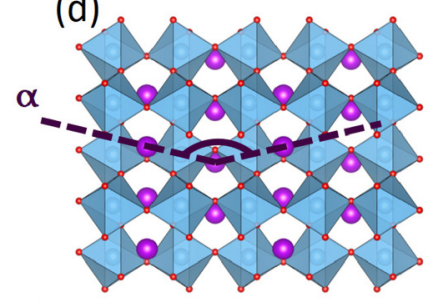

$\odot$

$<001>$
FIG. 1. HAADF images of the GTO crystal oriented along the $\langle 101\rangle$ direction (a) and the $\langle 001\rangle$ direction (b). Fast Fourier transform images are shown in the inset. Please note that some spatial drift is present. Bottom panels present the GTO crystal structure along the $\langle 101\rangle$ direction (c) and the $\langle 001\rangle$ directions (d).

\section{BULK GdTiO ${ }_{3}$ SAMPLES}

Bulk $\mathrm{GdTiO}_{3}$ (GTO) samples were obtained by the travelling floating-zone image method, Ref. [5]. They contained large, high-quality single-crystalline regions although superficial amounts of $\mathrm{Gd}_{2} \mathrm{Ti}_{2} \mathrm{O}_{7}$ and $\mathrm{TiO}_{2}$ could be detected by Raman microscopy consistent with the common overoxidation of $R \mathrm{TiO}_{3}$ sample surfaces [30,33]. Figure 1 shows the structural properties of one of such regions, characterized by means of high-angle annular dark field (HAADF) imaging and EELS using a JEOL JEM-ARM200cF scanning transmission electron microscope operated at $200 \mathrm{kV}$, and equipped with a probe aberration corrector and a Gatan Quantum EEL spectrometer. We present observations of the GTO crystal along the $\langle 101\rangle$ [Fig. 1(a)] and $\langle 001\rangle$ [Fig. 1(b)] directions. The fast Fourier transform images extracted from the same regions are shown in the insets and account for the orthorhombic symmetry. The tilting of the octahedral cage, corresponding to the complex pattern of the $P_{b n m}$ structure $\left(a^{-} a^{-} c^{+}\right.$in Glazer's notation) is sketched in Figs. 1(c) and 1(d). The atomic column coordinates were measured with subpixel resolution using an iterative process [34], looking for the center of mass of the Gd columns in the HAADF images of Figs. 1(a) and 1(b). The extracted averaged values of the deviation angles, 180- $\alpha$ and $180-\gamma$, are, respectively, $18^{\circ}$ and $13^{\circ}$. Based on these, we can extract the off-centering of the rare-earth ions to be around $0.25 \AA$ and conclude that the Ti-O-Ti angle is about $143.7^{\circ}$, close to other values reported in the literature $[4,5,8]$. We also acquired EELS spectra including the $\mathrm{Ti} L_{2,3}$ and $\mathrm{O} K$ edges on the bulk region. From these spectra we can estimate a relative Ti:O ratio of 1:3 using the routines implemented in the Digital Micrograph suite. This value is consistent with the nominal composition of GTO.

In line with previous reports on other RTO compounds $[16,33,35-39]$, we have found that the surface of GTO tends 

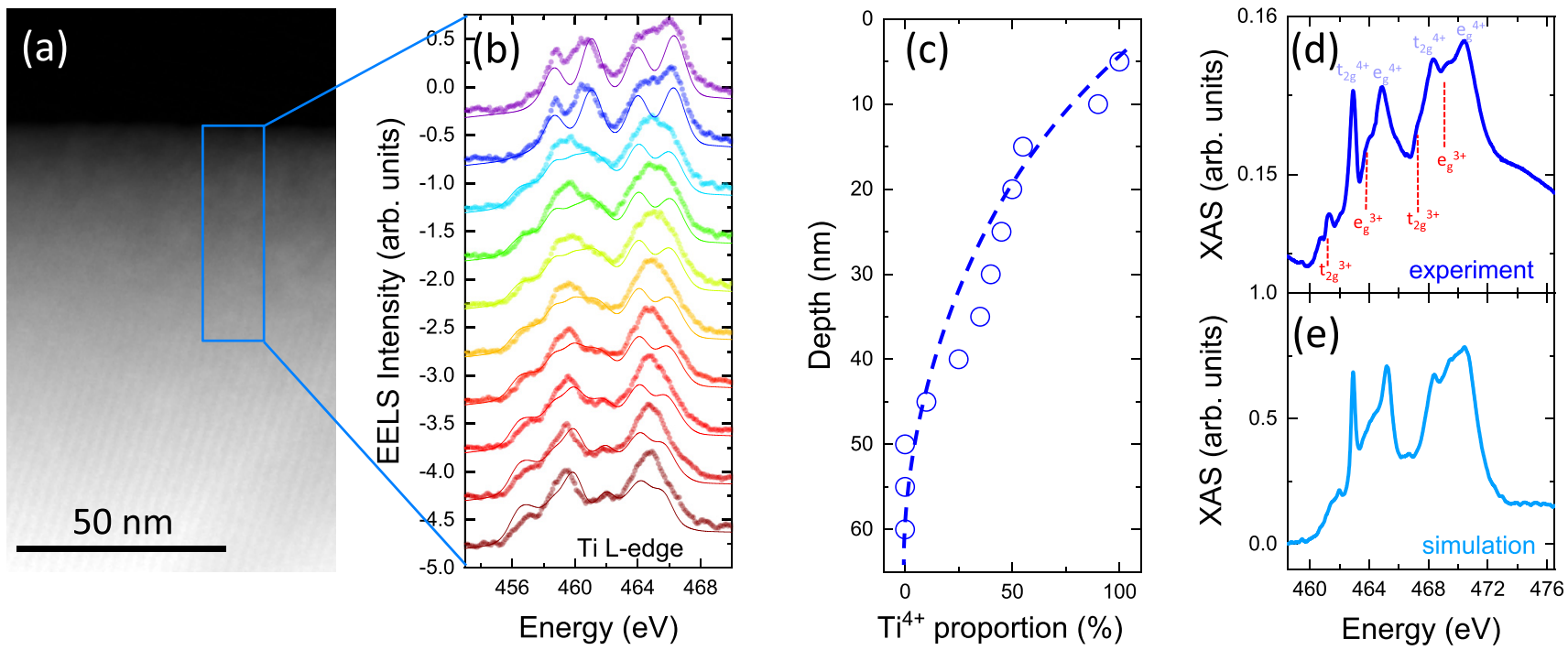

FIG. 2. (a) Low-magnification HAADF image of the GTO crystal edge. The blue rectangle represents the region where EEL spectra at the Ti $L_{2,3}$ edge (b) are extracted. The data are shown as symbols and in the fine lines are linear combination of simulations obtained from multiplet calculations. The proportion of $\mathrm{Ti}^{4+}$ is shown in (c). A XAS spectrum measured at $10 \mathrm{~K}$ is displayed in (d) and its simulation using experimental $3+$ and $4+$ spectra is presented in (e).

to be overoxidized. Figure 2 exhibits spectroscopic data for a region where this effect is particularly pronounced: EELS spectra were acquired as the electron beam was scanned from the specimen edge into the bulk. Figure 2(b) displays the Ti $L_{3,2}$ spectra down this line scan. A clear, progressive change in spectral shape is observed when comparing data from wellcrystallized GTO below the surface to more defective surface regions [cf. Fig. 2(a)]. The deepest regions show Ti $L_{3,2}$ spectra resembling for instance those acquired in XAS on $\mathrm{LaTiO}_{3}$ $[40,41]$ and can be well reproduced by simulations using the CTM4XAS software [42] (with a spectral broadening of $0.5 \mathrm{eV}$, accounting for the experimental resolution) and a pure $\mathrm{Ti}^{3+}$ state. The spectra taken in regions closer to the surface present similarities with data taken on $\mathrm{Ti}^{4+}$ compounds such as $\mathrm{SrTiO}_{3}$ [43] and with simulations for a pure $\mathrm{Ti}^{4+}$ state. Intermediate spectra can be mimicked by linear combinations of the simulations for $\mathrm{Ti}^{3+}$ and $\mathrm{Ti}^{4+}$, yielding estimates of the local $\mathrm{Ti}$ valence. The progressive variation of this $\mathrm{Ti}$ oxidation state across the observed area is plotted in Fig. 2(c). Remarkably, we also observed an evolution of the spectral shape of the $\mathrm{O} K$-edge prepeak (not shown), whose intensity is proportional to the number of holes in the hybridized Ti-O bond [29]. We could measure an enhancement of the prepeak intensity at the surface, consistent with a $\mathrm{Ti}$ valence closer to $4+$ and previous studies [39]. Earlier work on $\mathrm{NdTiO}_{3}$ has suggested that the deviation from $\mathrm{Ti}^{3+}$ to $\mathrm{Ti}^{4+}$ at the sample surface may be due to oxygen interstitials [30].

To complement this EELS analysis, we performed XAS in total electron yield (TEY) detection mode at the DEIMOS beam line $[44,45]$ of the synchrotron SOLEIL near Paris. The sample was cleaved in order to measure a fresh surface just before its introduction in the chamber that was then pumped to reach ultrahigh vacuum $\left(P \approx 10^{-10} \mathrm{mbar}\right)$. A XAS spectrum is shown in Fig. 2(d), after correction from a strong nonlinear background (probably due to sample charging) and normalization to the postedge intensity. The spectrum departs from the
$\mathrm{Ti}^{3+}$ EEL spectrum of Fig. 2(b) (bottom curves) or from the XAS measured in nearly stoichiometry $\mathrm{DyTiO}_{3}$ films [33]. As also found in, e.g., Ref. [35], this reflects some overoxidation of the sample surface after cleaving [Fig. 2(e)]. Comparing with previous EELS measurements in noncleaved samples, we can confidently state that the oxidation layer is here thinner $(\sim 2-3 \mathrm{~nm})$ than the probing depth $(\sim 9 \mathrm{~nm})$.

We now turn to the magnetic properties. Figures 3(a) and 3 (b) show the magnetization vs magnetic field and vs temperature, respectively. The saturation magnetization is lower than the value of $6 \mu \mathrm{B} / \mathrm{f} . u$. (f.u.: formula unit) expected for a collinear, ferrimagnetic arrangement of Ti and Gd with spinonly moment, as reported in Ref. [4]. This is probably due to the presence of impurity phases and to the imperfect oxygen stoichiometry, vide supra. The Curie temperature is about $25 \mathrm{~K}$, slightly lower than the expected $32 \mathrm{~K}$.

XAS was measured at $10 \mathrm{~K}$ for two opposite circular helicities. The XMCD was recorded from the difference between right [dark curves in Figs. 3(c) and 3(f)] and left [light curves in Figs. 3(c) and 3(f)] circularly polarized light at $45^{\circ}$ with respect to the normal incidence. The XMCD spectra are presented in Fig. 3(d) for the Ti $L_{3,2}$ edges and in Fig. 3(g) for the $\operatorname{Gd} M_{5,4}$ edges. We note that the shape of the Ti XMCD signal is probably affected by the nonstandard background correction, but is clearly different from that found at $\mathrm{SrTiO}_{3}$-based interfaces [46-48], suggesting that the mechanisms polarizing the Ti spins are different.

Remarkably, the XMCD signal at the Ti $L_{3,2}$ edges [Fig. 3(d)] shows a sign opposite to that reported by Cao et al. for $\mathrm{YTiO}_{3}$ thin films in which only $\mathrm{Ti}$ is magnetic [36], which suggests that in GTO the Ti moments align antiparallel to the external magnetic field. The XAS at the Gd $M_{5,4}$ edges [Fig. 3(f)] shows the typical shape for $\mathrm{Gd}^{3+}[49,50]$, with, however, a complex line shape at both edges. The XMCD [Fig. 3(g)] reaches about $25 \%$ of the total absorption, and is reminiscent of previous results on $\mathrm{Gd}^{3+}$ (e.g., in ferromag- 

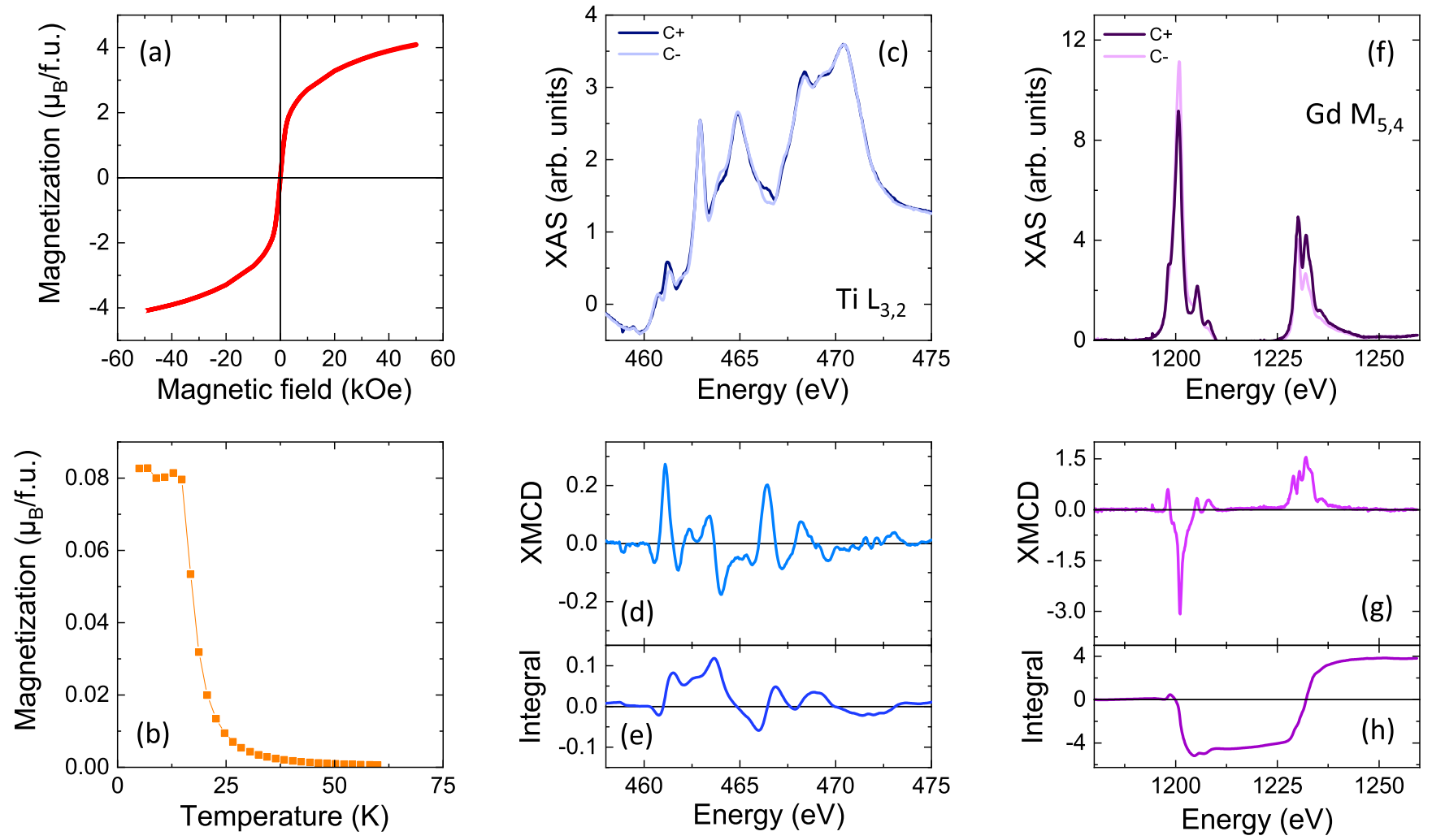

FIG. 3. (a) Magnetic field dependence of the magnetization at 4 K. (b) Temperature dependence of the magnetization measured with a field of $0.01 \mathrm{~T}$. XAS at the Ti $L_{3,2}$ (c) and Gd $M_{5,4}$ (f) edges recorded in total electron yield (TEY) for right (dark colors) and left (light colors) circularly polarized light. The incoming light is $45^{\circ}$, with respect to the normal incidence of the film. An in-plane magnetic field of $1 \mathrm{~T}$ is applied. Corresponding XMCD signals acquired at the Ti $L$ edge (d) and the Gd $M$ edges (g) with the associated integrated signal (e), (h).

netic $\mathrm{GdN}$ [51]). The negative sign at the $M_{5}$ edge is consistent with a $\mathrm{Gd}$ moment aligned parallel to the external magnetic field and therefore antiparallel to the Ti moment. Thus, our data provide a direct evidence of a ferrimagnetic spin order in $\mathrm{GdTiO}_{3}$.

The Ti and Gd XMCD spectra contain additional quantitative information concerning the magnetic structure of the titanate, such as the orbital and spin moments $m_{\text {orb }}$ and $m_{\text {spin }}$ that can be estimated by using the so-called "sum-rules" [52]. They are expressed as $m_{\text {spin }}=-(3 p-2 q)(N-n) / r$ and $m_{\text {orb }}=-2 q(N-n) / 3 r$ with $N=10$ for the $L_{3,2}$ edges and as $m_{\text {spin }}=-(5 p-3 q)(N-n) / 2 r$ and $m_{\text {orb }}=-q(N-n) / r$ with $N=14$ for the $M_{5,4}$ edges; $p$ is the integral of the XMCD of the first edge (e.g., $L_{2}$ or $\left.M_{4}\right), q$ is the total integral of the $\mathrm{XMCD}, r$ is the total integral of the XAS, and $n$ is the occupation number (i.e., $n=1$ for $\mathrm{Ti}^{3+}$ with $3 d^{1}$ configuration and $n=7$ for $\mathrm{Gd}^{3+}$ with $4 f^{7}$ configuration). In the case of $\mathrm{Ti}$ the large overlap between $\mathrm{Ti} L_{3}$ and $L_{2}$ edges complicates the determination of the spin moment [53,54]. However, from Fig. 3(e) $q \approx 0$, indicating the absence of an orbital moment on $\mathrm{Ti}$.

In the case of $\mathrm{Gd}$, the $M_{5}$ and $M_{4}$ edges are well separated and the sum rules can thus be applied. Unexpectedly, the integral of the XMCD for Gd is, however, not zero [Fig. 3(h)], which suggests the presence of a finite orbital moment, despite the nominally $4 f^{7}$ configuration of $\mathrm{Gd}^{3+}$. The reason is not clear at this stage, although it could be due to saturation effects artificially enhancing the signal at the $M_{5}$ edge [55]. It has also been suggested that $\mathrm{Gd}^{3+}$ may adopt a $4 f^{6} 5 d^{1}$ configuration [56], which would then lead to a nonzero orbital moment. We obtain $m_{\text {spin }}=1.91 \mu_{\mathrm{B}} / \mathrm{Gd}$ and $m_{\text {orb }}=-0.42 \mu_{\mathrm{B}} / \mathrm{Gd}$ at the measurement field of $1 \mathrm{~T}$. The spin and orbital moments appear to be antiparallel to each other, which would agree with a number of $f$ electrons lower than 7. The total Gd moment at $1 \mathrm{~T}$ is thus $1.49 \mu_{\mathrm{B}}$. Although we cannot reliably estimate $m_{\text {spin }}$ for Ti, we can assume the moment of Ti to be antiparallel to that of $\mathrm{Gd}$ (consistent with magnetization measurements [4,5,14,57-59]) and to be between 0.1 and $0.3 \mu_{\mathrm{B}}$ at that field. This would yield a total moment of about $1.2-1.4 \mu_{\mathrm{B}} /$ f.u., smaller than the value measured by SQUID magnetometry (2.7 $\mu \mathrm{B} /$ f.u. at $1 \mathrm{~T}$ ). This may be due to the inhomogeneity of the sample.

\section{GdTiO $_{3}$ THIN FILMS}

In order to study more intrinsic samples free from undesired phases, we turned to $\mathrm{GdTiO}_{3}$ thin films grown by pulsed laser deposition (PLD) on (001)-oriented $\mathrm{LaAlO}_{3}$ (LAO) substrates. Following the optimization of the PLD growth of $\mathrm{DyTiO}_{3}$ thin films [33], we set the growth temperature to $900^{\circ} \mathrm{C}$ and the growth pressure to $10^{-3}$ of pure Ar. Figure 4(a) displays a $2 \theta-\omega \mathrm{x}$-ray diffraction scan, attesting to the absence of impurity phases. Together with $\phi$ scans shown in Figs. 4(b) and 4(c) and the reflection high-energy electron 

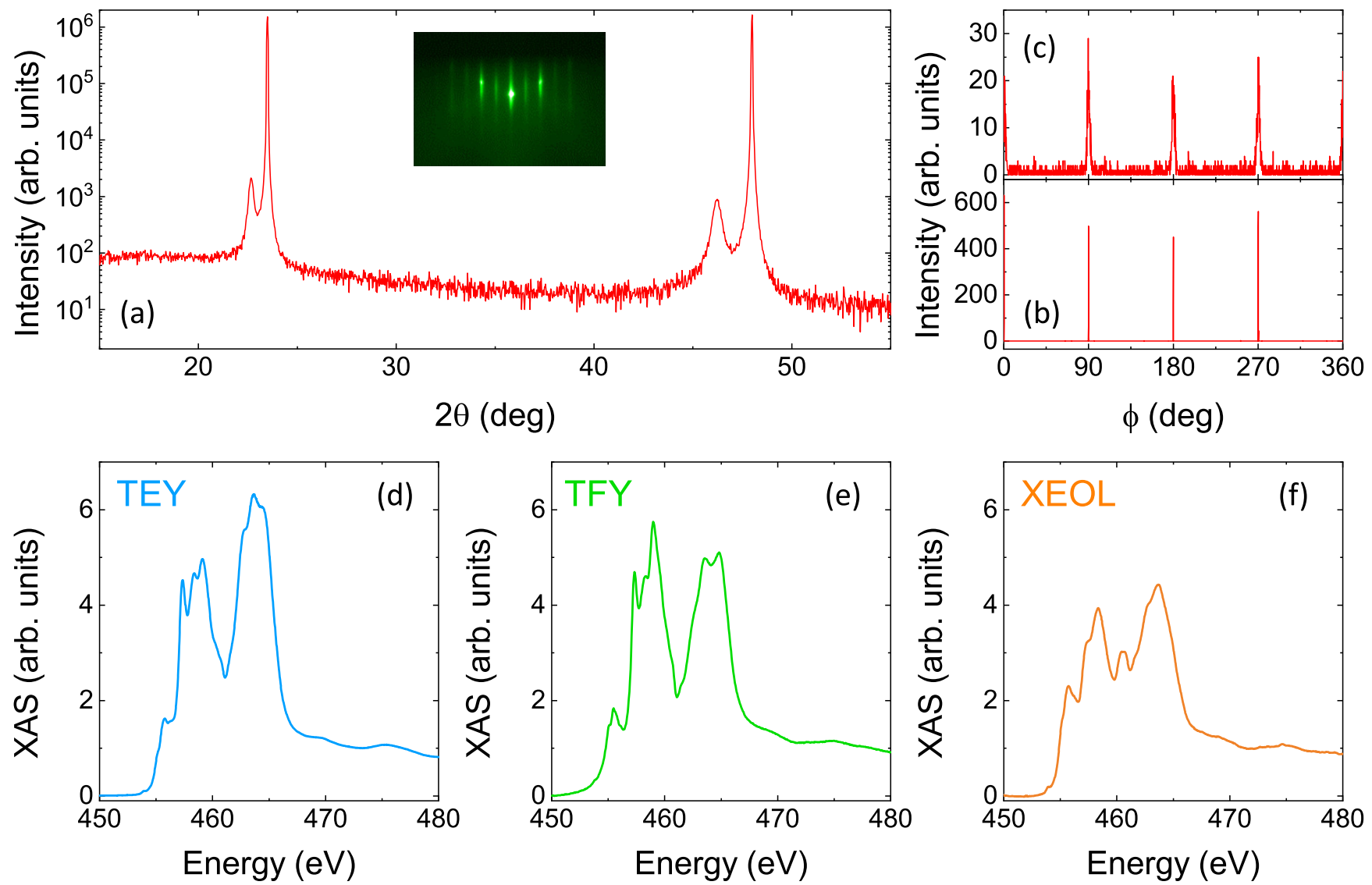

FIG. 4. (a) X-ray diffraction $2 \theta-\omega$ spectrum of a $60 \mathrm{~nm}$ GTO film grown on LAO(001). Inset: RHEED diagram at the end of the growth along a [100] pseudocubic direction. $\phi$ scans of the (202) reflections of LAO (b) and GTO (c) on the same sample. XAS at room temperature in TEY (d), TFY (e) and XEOL (f) in a 80-nm GTO film.

diffraction (RHEED) diagram [inset of Fig. 4(a)], these data indicate that the films are epitaxial. To characterize the $\mathrm{Ti}$ valence state, we used XAS at the Ti $L_{3,2}$ edges at the XTreme beamline [60] of the Swiss Light Source in three detection modes: TEY, total fluorescence yield (TFY) and x-ray excited optical luminescence (XEOL). While TEY only probes the first nm of the sample, TFY typically probes a few tens of $\mathrm{nm}$ and XEOL is a transmission technique probing the whole film thickness [61]. The data are presented in Figs. 4(d)-4(f) and show that, in contrast to the case of the bulk sample, even in TEY the Ti spectrum indicates a large amount of $\mathrm{Ti}^{3+}$. The $\mathrm{Ti}^{3+}$ content increases when probing a larger thickness using TFY, and appears to approach 100\% in XEOL. These results attest to the good quality of our GTO thin films.

Figure 5 presents the magnetic characterization of these samples. Figures 5(a) and 5(b) show magnetization as a function of magnetic field and temperature, respectively. The magnetization at the highest field (5 T) approaches $6 \mu_{\mathrm{B}} / \mathrm{f}$.u., the value expected for a ferrimagnetic state with a spin only moment of $1 \mu_{\mathrm{B}}$ for $\mathrm{Ti}$ and $7 \mu_{\mathrm{B}}$ for $\mathrm{Gd}$. The $T_{\mathrm{C}}$ is around $25 \mathrm{~K}$ as for the bulk sample [Fig. 3(b)] and a bit lower than the expected value of $32 \mathrm{~K}$. Figures $5(\mathrm{c})$ and $5(\mathrm{f})$ show Ti $L_{3,2}$ XAS spectra measured in TFY and XEOL, respectively, for two opposite light helicities. Unlike for the GTO bulk sample, the spectra only had to be corrected for a linear background and were normalized to the postedge amplitude. The corre- sponding XMCD spectra are shown in Figs. 5(d) and 5(g). In both cases a clear signal is obtained, with maximum values approaching $25 \%$. To the best of our knowledge, this is the largest XMCD reported at the Ti $L_{3,2}$ edge. The XMCD in TFY and XEOL show similarities between each other [and also with the data for bulk GTO shown in Figs. 3(d) and 3(e)], but also distinct features, possibly reflecting a proximity-induced dichroism of the $\mathrm{Ti}^{4+}$ ions present near the sample surface, probed in higher concentration in TFY compared to XEOL. Figures 5(i) and 5(j) show the XAS and XMCD simulations for a $\mathrm{Ti}^{3+}$ ion. The simulations are obtained using the MultiX code [62]. The crystal field splitting is calculated starting from a structure file of the $\mathrm{GdTiO}_{3}$ structure obtained by DFT. The $3 d$ spin-orbit coupling was set to zero. The XMCD shows good agreement with the measured data. This simulation, which has a similar magnitude as the measured data, corresponds to a Ti magnetic moment of $0.49 \mu_{\mathrm{B}}$.

\section{DFT SIMULATIONS}

To confirm our experimental results, we have performed density functional theory (DFT) simulations on $\mathrm{GdTiO}_{3}$ both in bulk and film form. Gd $4 f$ electrons are explicitly treated in the simulations. We have used the PBESOL functional with an on-site potential $U=2.5 \mathrm{eV}$ on Ti $3 d$ levels [11] in order 

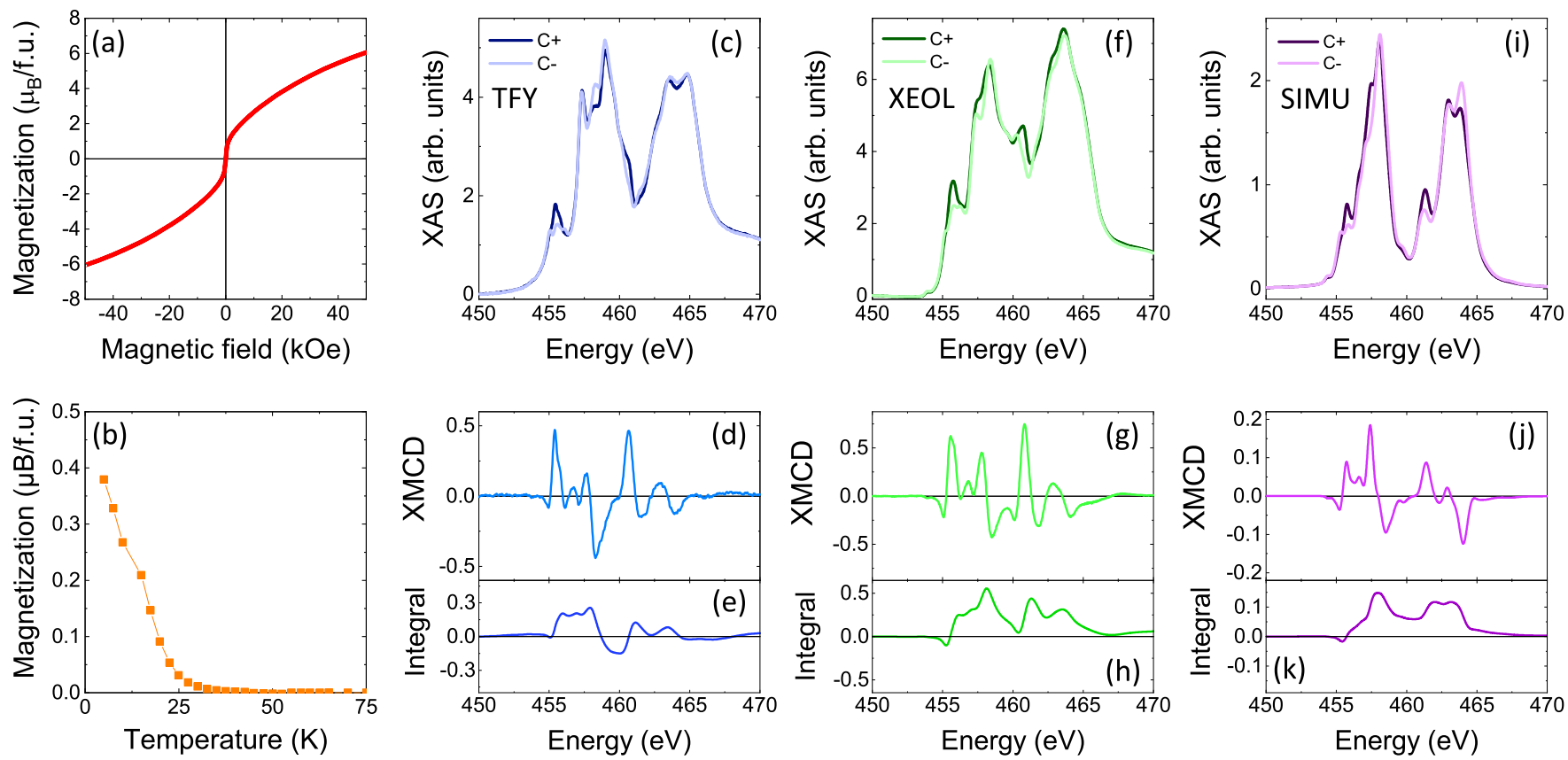

FIG. 5. (a) Magnetic field dependence of the magnetization at $5 \mathrm{~K}$. (b) Temperature dependence of the magnetization in a field of $0.1 \mathrm{~T}$. XAS at $5 \mathrm{~T}(\mathrm{c}), \mathrm{XMCD}(\mathrm{d})$ and XMCD integral (e) at the Ti $L_{3,2}$ edge measured in TFY. XAS at $5 \mathrm{~T}$ (f), XMCD (g) and XMCD integral (h) at the Ti $L_{3,2}$ edge measured in XEOL. (i) XAS and (j) XMCD simulations for a $\mathrm{Ti}^{3+}$ ion in the GTO structure using the MultiX code. (k) Simulated XMCD integral.

to amend self-interaction errors (no correction is needed on Gd $4 f$ states since these levels are half-filled). Structural relaxations using G-type AFM and FM orders on Ti sublattices and FM, A-type, C-type and G-type AFM orders on $\mathrm{Gd}^{3+}$ cations were performed until forces are lower than $1 \mathrm{meV} / \AA$ with a $k$-point mesh of $6 * 6 * 4(4 * 4 * 4)$ for bulk (film) structure. Spin-orbit interaction was added after identification of the lowest energy state.

The bulk material relaxes to an orthorhombic Pbnm structure $\left(a^{-} a^{-} c^{+}\right.$rotation pattern in Glazer's notation) with lattice parameters evaluated to $5.376,5.754$, and $7.665 \AA$, yielding less than $0.3 \%$ of volume error with respect to experiments [5]. The material is also correctly found insulating with a band gap of $0.920 \mathrm{eV}$. Just as found in experiments, the lowest energy state is reached when each magnetic sublattice orders ferromagnetically but with antiparallel spins between Ti $\left(0.88 \mu_{\mathrm{B}}\right)$ and $\mathrm{Gd}\left(6.81 \mu_{\mathrm{B}}\right)$ moments (resultant magnetic moment of $24 \mu_{\mathrm{B}}$ per orthorhombic Pbnm cell). Once we include the spin-orbit interaction, although restricting the spins to be aligned along the $c^{+}$rotation axis, we do not identify any substantial orbital moment on $\mathrm{Ti}$ and Gd (only $0.03 \mu_{\mathrm{B}}$ and $0.07 \mu_{\mathrm{B}}$ for $\mathrm{Ti}^{3+}$ and $\mathrm{Gd}^{3+}$ ions, respectively). We have also considered the strain effect imposed by the $\mathrm{LaAlO}_{3}$ substrate. Due to the compressive strain imposed by the substrate [63], the symmetry of the material is lowered to a $P 2_{1} / m$ structure with the in phase rotation aligned along the substrate $\left(a^{+} a^{-} c^{-}\right.$ rotation pattern) so as to minimize the constraint experienced by the film. This finding is in line with previous DFT simulations [37]. However, the strain does not affect the magnetic structure $\left(\mu_{\mathrm{Ti}}=0.87 \mu_{\mathrm{B}}\right.$ and $\left.\mu_{\mathrm{Gd}}=6.76 \mu_{\mathrm{B}}\right)$ and the resulting orbital moment on $\mathrm{Ti}\left(0.002 \mu_{\mathrm{B}}\right)$ and $\mathrm{Gd}\left(0.07 \mu_{\mathrm{B}}\right)$ atoms.

\section{SUMMARY}

In summary, we have performed a detailed structural and magnetic characterization of $\mathrm{GdTiO}_{3}$ in both bulk and thin film form using a combination of advanced techniques. Using XAS and EELS, we found that in both types of samples the Ti valence deviates from the nominal $3+$ to $4+$ at the surface (but much more so in uncleaved bulk samples). This is consistent with earlier reports on rare-earth titanates. We performed XMCD measurements at the $\mathrm{Ti} L_{3,2}$ and $\mathrm{Gd} M_{5,4}$ edges and found large dichroic signals. The sign of the XMCD signals provide direct evidence for a ferrimagnetic spin order. In thin films, the Ti XMCD reaches $25 \%$ when probing almost purely $3+\mathrm{Ti}$ (in total fluorescence yield or in transmission). The integral of the Ti XMCD is vanishingly small in both bulk and thin film GTO, indicating the absence of measurable orbital moment on $\mathrm{Ti}$, as also found in $\mathrm{LaTiO}_{3}$ and $\mathrm{YTiO}_{3}$. Our work calls for further studies of the Ti spin and orbital moment in other rare-earth titanates with more complex, noncollinear spin structures.

\section{ACKNOWLEDGMENTS}

We acknowledge fruitful discussions with P. Sainctavit and M.-A. Arrio. Research at CNRS/Thales was supported by the ERC Consolidator Grant "MINT" (Contract No. 615759). We thank Synchrotron SOLEIL for the provision of beam time at DEIMOS (Proposal No. 20140497). Electron microscopy observations were carried out at the Centro Nacional de Microscopía Electronica at the Universidad Complutense de Madrid. Financial support from the Spanish MCINN 
RTI2018-097895-B-C43, MAT2017-87134-C02 and RTI2018-099054-J-I00 is also acknowledged (UCM). We also acknowledge financial support from the Deutsche Forschungsgemeinschaft (DFG) through CRC 1238 (Project
No. 277146847, subproject A02) and access granted to HPC ressources of Criann through Projects No. 2020005 and No. 2007013 and of Cines through the DARI Project No. A0080911453).
[1] J. B. Goodenough, Theory of the role of covalence in the perovskite-type manganites [La, $M(I I)] \mathrm{MnO}_{3}$, Phys. Rev. 100, 564 (1955).

[2] M. Mochizuki and M. Imada, Orbital physics in the perovskite Ti oxides, New J. Phys. 6, 154 (2004).

[3] C. Ulrich, G. Khaliullin, S. Okamoto, M. Reehuis, A. Ivanov, H. He, Y. Taguchi, Y. Tokura, and B. Keimer, Magnetic Order and Dynamics in An Orbitally Degenerate Ferromagnetic Insulator, Phys. Rev. Lett. 89, 167202 (2002).

[4] H. D. Zhou and J. B. Goodenough, Localized or itinerant $\mathrm{TiO}_{3}$ electrons in $\mathrm{RTiO}_{3}$ perovskites, J. Phys. Condens. Matter 17, 7395 (2005).

[5] A. C. Komarek, H. Roth, M. Cwik, W.-D. Stein, J. Baier, M. Kriener, F. Bourée, T. Lorenz, and M. Braden, Magnetoelastic coupling in $R \mathrm{TiO}_{3}(R=\mathrm{La}, \mathrm{Nd}, \mathrm{Sm}, \mathrm{Gd}, \mathrm{Y})$ investigated with diffraction techniques and thermal expansion measurements, Phys. Rev. B 75, 224402 (2007).

[6] J. E. Greedan, The rare earth-titanium (III) perovskite oxidesan isostructural series with a remarkable variation in physical properties, J. Less-Common Met. 111, 335 (1985).

[7] T. Katsufuji, Y. Taguchi, and Y. Tokura, Transport and magnetic properties of a Mott-Hubbard system whose bandwidth and band filling are both controllable: $\mathrm{R} 1-\mathrm{XCaxTiO}_{3}+\mathrm{y} / 2$, Phys. Rev. B 56, 10145 (1997).

[8] M. Cwik, T. Lorenz, J. Baier, R. Müller, G. André, F. Bourée, F. Lichtenberg, A. Freimuth, R. Schmitz, E. Müller-Hartmann, and M. Braden, Crystal and magnetic structure of $\mathrm{LaTiO}_{3}$ : Evidence for nondegenerate $t_{2 g}$ orbitals, Phys. Rev. B 68, 060401(R) (2003).

[9] M. Mochizuki and M. Imada, Origin of G-type antiferromagnetism and orbital-spin structures in $\mathrm{LaTiO}_{3}$, J. Phys. Soc. Jpn. 70, 2872 (2001).

[10] J. Akimitsu, H. Ichikawa, N. Eguchi, T. Miyano, M. Nishi, K. Kakurai, N. Di, J. A. Kimitsu, H. I. Chikawa, N. E. Guchi, J. Akimitsu, H. Ichikawa, N. Eguchi, T. Miyano, M. Nishi, K. Kakurai, N. Di, J. A. Kimitsu, H. I. Chikawa, and N. E. Guchi, Direct observation of orbital ordering in $\mathrm{YTiO}_{3}$ by means of the polarized neutron diffraction technique, J. Phys. Soc. Jpn. 70, 3475 (2001)

[11] J. Varignon, M. N. Grisolia, D. Preziosi, P. Ghosez, and M. Bibes, Origin of the orbital and spin ordering in rare-earth titanates, Phys. Rev. B 96, 235106 (2017).

[12] J. Varignon, M. Bibes, and A. Zunger, Origin of band gaps in 3 $d$ perovskite oxides, Nat. Commun. 10, 1658 (2019).

[13] V. M. Goldschmidt, Die gesetze der krystallochemie, Naturwissenschaften 14, 477 (1926).

[14] G. Amow, J.-S. Zhou, and J. B. Goodenough, Peculiar magnetism of the $\mathrm{Sm}_{(1-\mathrm{x})} \mathrm{Gd}_{\mathrm{x}} \mathrm{TiO}_{3}$ system, J. Solid State Chem. 154, 619 (2000).

[15] E. Pavarini, S. Biermann, A. Poteryaev, A. I. Lichtenstein, A. Georges, and O. K. Andersen, Mott Transition and Suppression of Orbital Fluctuations in Orthorhombic $3 d 1$ Perovskites, Phys. Rev. Lett. 92, 176403 (2004).
[16] F. Iga, M. Tsubota, M. Sawada, H. B. Huang, S. Kura, M. Takemura, K. Yaji, M. Nagira, A. Kimura, T. Jo, T. Takabatake, H. Namatame, and M. Taniguchi, Determination of the Orbital Polarization in $\mathrm{YTiO}_{3}$ by Using Soft X-Ray Linear Dichroism, Phys. Rev. Lett. 93, 257207 (2004).

[17] I. V. Solovyev, Lattice distortion and magnetism of $3 d-t_{2 g}$ perovskite oxides, Phys. Rev. B 74, 054412 (2006).

[18] G. Khaliullin and S. Okamoto, Theory of orbital state and spin interactions in ferromagnetic titanates, Phys. Rev. B 68, 205109 (2003).

[19] S. Okatov, A. Poteryaev, and A. Lichtenstein, Structural distortions and orbital ordering in $\mathrm{LaTiO}_{3}$ and $\mathrm{YTiO}_{3}$, Europhysics Letters (EPL) 70, 499 (2005).

[20] T. Kiyama and M. Itoh, Presence of $3 d$ Quadrupole Moment in $\mathrm{LaTiO}_{3}$ Studied by ${ }^{47,49}$ Ti NMR, Phys. Rev. Lett. 91, 167202 (2003).

[21] G. I. Meijer, W. Henggeler, J. Brown, O.-S. Becker, J. G. Bednorz, C. Rossel, and P. Wachter, Reduction of ordered moment in strongly correlated $\mathrm{LaTiO}_{3+\delta}$ upon band filling, Phys. Rev. B 59, 11832 (1999).

[22] M. Tsubota, F. Iga, T. Takabatake, N. Kikugawa, T. Suzuki, I. Oguro, H. Kawanaka, and H. Bando, Low-field magnetic anisotropy in Mott-insulating ferromagnet $\mathrm{Y}_{1-x} \mathrm{Ca}_{\mathrm{x}} \mathrm{TiO}_{3}$ $(x \leqslant 1.0)$, Physica B 281-282, 622 (2000).

[23] Y. Okimoto, T. Katsufuji, Y. Okada, T. Arima, and Y. Tokura, Optical spectra in $(\mathrm{La}, \mathrm{Y}) \mathrm{TiO}_{3}$ : Variation of Mott-Hubbard gap features with change of electron correlation and band filling, Phys. Rev. B 51, 9581 (1995).

[24] T. Katsufuji and Y. Tokura, Anomalous variation of phonon raman intensities near the metal-to-Mott-insulator transition in titanium oxide systems, Phys. Rev. B 50, 2704 (1994).

[25] Y. Tokura, Y. Taguchi, Y. Okada, Y. Fujishima, T. Arima, K. Kumagai, and Y. Iye, Filling Dependence of Electronic Properties on the Verge of Metal-Mott-Insulator Transition in $\mathrm{Sr}_{1}-\mathrm{xLa}_{\mathrm{x}} \mathrm{TiO}_{3}$, Phys. Rev. Lett. 70, 2126 (1993).

[26] P. Moetakef, J. Y. Zhang, A. Kozhanov, B. Jalan, R. Seshadri, S. J. Allen, and S. Stemmer, Transport in ferromagnetic $\mathrm{GdTiO}_{3} / \mathrm{SrTiO}_{3}$ heterostructures, Appl. Phys. Lett. 98, 112110 (2011).

[27] P. Moetakef, J. R. Williams, D. G. Ouellette, A. P. Kajdos, D. Goldhaber-Gordon, S. J. Allen, and S. Stemmer, Carrier-Controlled Ferromagnetism in $\mathrm{SrTiO}_{3}$, Phys. Rev. X 2, 021014 (2012).

[28] D. G. Ouellette, P. Moetakef, T. A. Cain, J. Y. Zhang, S. Stemmer, D. Emin, and S. J. Allen, High-density twodimensional small polaron gas in a delta-doped mott insulator, Sci. Rep. 3, 3284 (2013).

[29] M. N. Grisolia, J. Varignon, G. Sanchez-Santolino, A. Arora, S. Valencia, M. Varela, R. Abrudan, E. Weschke, E. Schierle, J. E. Rault, J.-P. Rueff, A. Barthélémy, J. Santamaria, and M. Bibes, Hybridization-controlled charge transfer and induced magnetism at correlated oxide interfaces, Nat. Phys. 12, 484 (2016). 
[30] P. Xu, Y. Ayino, C. Cheng, V. S. Pribiag, R. B. Comes, P. V. Sushko, S. A. Chambers, and B. Jalan, Predictive Control over Charge Density in the Two-Dimensional Electron Gas at the Polar-Nonpolar $\mathrm{NdTiO}_{3} / \mathrm{SrTiO}_{3}$ Interface, Phys. Rev. Lett. 117, 106803 (2016).

[31] S. Raghavan, J. Y. Zhang, S. Stemmer, Two-Dimensional Electron Liquid at the (111) $\mathrm{SmTiO}_{3} / \mathrm{SrTiO}_{3}$ Interface, Appl. Phys. Lett. 106, 132104 (2015).

[32] Y. Cao, Z. Yang, M. Kareev, X. Liu, D. Meyers, S. Middey, D. Choudhury, P. Shafer, J. Guo, J. W. Freeland, E. Arenholz, L. $\mathrm{Gu}$, and $\mathrm{J}$. Chakhalian, Magnetic Interactions at the Nanoscale in Trilayer Titanates, Phys. Rev. Lett. 116, 076802 (2016).

[33] R. Aeschlimann, D. Preziosi, P. Scheiderer, M. Sing, S. Valencia, J. Santamaria, C. Luo, H. Ryll, F. Radu, R. Claessen, C. Piamonteze, and M. Bibes, A living-dead magnetic layer at the surface of ferrimagnetic $\mathrm{DyTiO}_{3}$ thin films, Adv. Mater. 30, 1707489 (2018).

[34] Y. Gong, Z. Liu, A. R. Lupini, G. Shi, J. Lin, S. Najmaei, Z. Lin, A. L. Elías, A. Berkdemir, G. You, H. Terrones, M. Terrones, R. Vajtai, S. T. Pantelides, S. J. Pennycook, J. Lou, W. Zhou, and P. M. Ajayan, Band gap engineering and layer-by-layer mapping of selenium-doped molybdenum disulfide, Nano Lett. 14, 442 (2014).

[35] C. Ulrich, G. Ghiringhelli, A. Piazzalunga, L. Braicovich, N. B. Brookes, H. Roth, T. Lorenz, and B. Keimer, Orbital excitations in $\mathrm{YTiO}_{3}$ and $\mathrm{LaTiO}_{3}$ probed by resonant inelastic soft x-ray scattering, Phys. Rev. B 77, 113102 (2008).

[36] Y. Cao, P. Shafer, X. Liu, D. Meyers, M. Kareev, S. Middey, J. W. Freeland, E. Arenholz, and J. Chakhalian, Magnetism and electronic structure of $\mathrm{YTiO}_{3}$ thin films, Appl. Phys. Lett. 107, 112401 (2015).

[37] M. N. Grisolia, F. Y. Bruno, D. Sando, H. J. Zhao, E. Jacquet, X. M. Chen, L. Bellaiche, A. Barthélémy, and M. Bibes, Structural, magnetic, and electronic properties of $\mathrm{GdTio}_{3}$ Mott insulator thin films grown by pulsed laser deposition, Appl. Phys. Lett. 105, 172402 (2014).

[38] A. Ohtomo, D. A. Muller, J. L. Grazul, and H. Y. Hwang, Epitaxial growth and electronic structure of $\mathrm{LaTiO}_{x}$ films, Appl. Phys. Lett. 80, 3922 (2002).

[39] N. N. Kovaleva, A. V. Boris, P. Yordanov, A. Maljuk, E. Brücher, J. Strempfer, M. Konuma, I. Zegkinoglou, C. Bernhard, A. M. Stoneham, and B. Keimer, Optical response of ferromagnetic $\mathrm{YTiO}_{3}$ studied by spectral ellipsometry, Phys. Rev. B 76, 155125 (2007).

[40] M. Abbate, F. M. F. de Groot, J. C. Fuggle, A. Fujimori, Y. Tokura, Y. Fujishima, O. Strebel, M. Domke, G. Kaindl, J. van Elp, B. T. Thole, G. A. Sawatzky, M. Sacchi, and N. Tsuda, Soft-x-ray-absorption studies of the location of extra charges induced by substitution in controlled-valence materials, Phys. Rev. B 44, 5419 (1991).

[41] M. W. Haverkort, Z. Hu, A. Tanaka, G. Ghiringhelli, H. Roth, M. Cwik, T. Lorenz, C. Schüssler-Langeheine, S. V. Streltsov, A. S. Mylnikova, V. I. Anisimov, C. de Nadai, N. B. Brookes, H. H. Hsieh, H.-J. Lin, C. T. Chen, T. Mizokawa, Y. Taguchi, Y. Tokura, D. I. Khomskii, and L. H. Tjeng, Determination of the Orbital Moment and Crystal-Field Splitting in $\mathrm{LaTiO}_{3}$, Phys. Rev. Lett. 94, 056401 (2005).

[42] E. Stavitski, F. M. F. De Groot, F. M. F. de Groot, and F. M. F. De Groot, The CTM4XAS program for eels and xas spectral shape analysis of transition metal L edges, Micron 41, 687 (2010).

[43] H. W. Jang, D. A. Felker, C. W. Bark, Y. Wang, M. K. Niranjan, C. T. Nelson, Y. Zhang, D. Su, C. M. Folkman, S. H. Baek, S. Lee, K. Janicka, Y. Zhu, X. Q. Pan, D. D. Fong, E. Y. Tsymbal, M. S. Rzchowski, and C. B. Eom, Metallic and insulating oxide interfaces controlled by electronic correlations, Science 331, 886 (2011).

[44] P. Ohresser, E. Otero, F. Choueikani, K. Chen, S. Stanescu, F. Deschamps, T. Moreno, F. Polack, B. Lagarde, J. P. Daguerre, F. Marteau, F. Scheurer, L. Joly, J. P. Kappler, B. Muller, O. Bunau, and P. Sainctavit, DEIMOS: A beamline dedicated to dichroism measurements in the $350-2500 \mathrm{eV}$ energy range, Rev. Sci. Instrum. 85, 013106 (2014).

[45] L. Joly, E. Otero, F. Choueikani, F. Marteau, L. Chapuis, and P. Ohresser, Fast continuous energy scan with dynamic coupling of the monochromator and undulator at the DEIMOS beamline, J. Synchrotron. Rad. 21, 502 (2014).

[46] J. Garcia-Barriocanal, J. C. Cezar, F. Y. Bruno, P. Thakur, N. B. Brookes, C. Utfeld, A. Rivera-Calzada, S. R. Giblin, J. W. Taylor, J. A. Duffy, S. B. Dugdale, T. Nakamura, K. Kodama, C. Leon, S. Okamoto, and J. Santamaria, Spin and orbital Ti magnetism at $\mathrm{LaMnO}_{3} / \mathrm{SrTiO}_{3}$ interfaces, Nat. Commun. 1, 82 (2010).

[47] J.-S. Lee, Y. W. Xie, H. K. Sato, C. Bell, Y. Hikita, H. Y Hwang, and C.-C. Kao, Titanium dxy ferromagnetism at the $\mathrm{LaAlO}_{3} / \mathrm{SrTiO}_{3}$ interface, Nat. Mater. 12, 703 (2013).

[48] M. Salluzzo, S. Gariglio, D. Stornaiuolo, V. Sessi, S. Rusponi, C. Piamonteze, G. M. De Luca, M. Minola, D. Marré, A. Gadaleta, H. Brune, F. Nolting, N. B. Brookes, and G. Ghiringhelli, Origin of Interface Magnetism in $\mathrm{BiMnO}_{3} / \mathrm{SrTiO}_{3}$ and $\mathrm{LaAlO}_{3} / \mathrm{SrTiO}_{3}$ Heterostructures, Phys. Rev. Lett. 111, 087204 (2013).

[49] J. B. Goedkoop, B. T. Thole, G. van der Laan, G. A. Sawatzky, F. M. F. de Groot, and J. C. Fuggle, Calculations of magnetic $\mathrm{X}$-ray dichroism in the $3 d$ absorption spectra of rare-earth compounds, Phys. Rev. B 37, 2086 (1988).

[50] K. Ugendar, V. Hari Babu, V. Raghavendra Reddy, and G. Markaneyulu, Cationic ordering and magnetic properties of rare-earth doped $\mathrm{NiFe}_{2} \mathrm{O}_{4}$ probed by mössbauer and x-ray spectroscopies, J. Magn. Magn. Mater. 484, 291 (2019).

[51] F. Leuenberger, A. Parge, W. Felsch, K. Fauth, and M. Hessler, GdN thin films: Bulk and local electronic and magnetic properties, Phys. Rev. B 72, 014427 (2005).

[52] C. T. Chen, Y. U. Idzerda, H. J. Lin, N. V. Smith, G. Meigs, E. Chaban, G. H. Ho, E. Pellegrin, and F. Sette, Experimental Confirmation of the X-Ray Magnetic Circular Dichroism Sum Rules for Iron and Cobalt, Phys. Rev. Lett. 75, 152 (1995).

[53] A. Scherz, H. Wende, K. Baberschke, J. Minar, D. Benea, and $\mathrm{H}$. Ebert, Relation between $L_{2,3} \mathrm{XMCD}$ and the magnetic ground-state properties for the early $3 d$ element V, Phys. Rev. B 66, 184401 (2002).

[54] A. Scherz, H. Wende, C. Sorg, K. Baberschke, J. Min, J. Minr, D. Benea, and H. Ebert, Limitations of integral XMCD sumrules for the early $3 d$ elements, Phys. Scr. T115, 586 (2005).

[55] R. Nakajima, J. Stöhr, and Y. U. Idzerda, Electron-yield saturation effects in $L$-edge X-ray magnetic circular dichroism spectra of Fe, Co, and Ni, Phys. Rev. B 59, 6421 (1999).

[56] S. Abdelouahed, N. Baadji, and M. Alouani, Electronic structure and $\mathrm{x}$-ray magnetic circular dichroism of gadolinium 
beyond the local spin density approximation, Phys. Rev. B 75, 094428 (2007).

[57] C. W. Turner, M. F. Collins, and J. E. Greedan, Magnetic structures of the rare earth orthotitanites $\mathrm{RTio}_{3} ; \mathrm{R}=\mathrm{Tb}, \mathrm{Dy}, \mathrm{Tm}, \mathrm{Yb}$, J. Magn. Magn. Mater. 23, 265 (1981).

[58] C. W. Turner and J. E. Greedan, Ferrimagnetism in the rare earth titanium (III) Oxides, $\mathrm{RTiO}_{3} ; \mathrm{R}=\mathrm{Gd}, \mathrm{Tb}, \mathrm{Dy}, \mathrm{Ho}, \mathrm{Er}, \mathrm{Tm}$, J. Solid State Chem. 34, 207 (1980).

[59] J. P. Goral and J. E. Greedan, Magnetic behavior in the system $\mathrm{La}_{x} \mathrm{Gd}_{1-x} \mathrm{TiO}_{3}$, J. Solid State Chem. 43, 204 (1982).

[60] C. Piamonteze, U. Flechsig, S. Rusponi, J. Dreiser, J. Heidler, M. Schmidt, R. Wetter, M. Calvi, T. Schmidt, H. Pruchova, J. Krempasky, C. Quitmann, H. Brune, and F. Nolting, X-treme beamline at SLS: X-ray magnetic circular and linear dichroism at high field and low temperature, J. Synchrotron Radiat. 19, 661 (2012).

[61] D. J. Huang, C. F. Chang, J. Chen, H. J. Lin, S. C. Chung, H. T. Jeng, G. Y. Guo, W. B. Wu, S. G. Shyu, and C. T. Chen, Orbital moments of $\mathrm{CrO}_{2}$ and $\mathrm{Fe}_{3} \mathrm{O}_{4}$ studied by MCD in soft Xray absorption, J. Electron Spectrosc. Relat. Phenom. 137-140, 633 (2004).

[62] A. Uldry, F. Vernay, and B. Delley, Systematic computation of crystal-field multiplets for X-ray core spectroscopies, Phys. Rev. B 85, 125133 (2012).

[63] J. M. Rondinelli and N. A. Spaldin, Structure and properties of functional oxide thin films: Insights from electronic-structure calculations, Adv. Mater. 23, 3363 (2011). 